\title{
Quête de soi et dépression chez Albert Memmi
}

\section{Chahinaz Sabri Mohamed}

(Assistante au département de français- Faculté de pédagogie)

\section{Résumé}

Originaire de Tunisie, issu d'une famille pauvre de langue maternelle arabe, Albert Memmi a vécu à Paris depuis 1950. La plupart de ses œuvres se déroulent en Tunisie mettant en scène des personnages qui aspirent à se réaliser et à retrouver leur soi. Albert Memmi est sociologue et romancier. Son œuvre fonde la littérature tunisienne et maghrébine. Il est considéré comme l'une des figures emblématiques de la lutte anticoloniale.

En effet, les œuvres de Memmi qui sont l'objet de notre thèse incarne bien la quête de soi. Avec «La Statue de Sel» et «Le Pharaon », Memmi éclaircit le problème que tout être affronte : celui de s'affirmer et de trouver un sens à son existence. Dans ses œuvres, paraît une quête espérée par notre romancier que par ses créateurs.

Bien entendu, la quête de soi fait partie de la vie humaine et sans doute elle restera toujours un élément inséparable de notre existence. Depuis sa naissance, l'être tente de construire une vue sur lui-même ainsi que sur autrui. Bien que la quête de soi soit considérée comme le fil d'Ariane aboutissant vers un semblant de voie recouvert des difficultés, elle demeure comme une lumière au firmament de notre existence.

Dans cette recherche, nous allons étudier le schéma quinaire de Greimas et Larivaille en mettant l'accent sur les motifs qui ont incité les personnages à s'engager dans cette quête comme le sentiment de la fragmentation, le sentiment d'être étranger, le sentiment de la solitude et enfin le sentiment de la dépossession. Nous nous proposons aussi de montrer les manifestations de la 
Quête de soi et dépression chez Albert Memmi

quête et les tentatives de la reconquête de soi. La dernière phase de cette étude comportera les répercussions et les conséquences de cette quête, à savoir le sort des personnages.

Mots clés : quête de soi- tentatives-dépression 


\section{Chahinaz Sabri Mohamed}

\section{البحث عن الذات والاكتئاب}

\section{شاهيناز صبري محد}

ملخص - - م

عاش البير ميمي الكاتب التونسي، الذي انحدر من عائلة عربية فقيرة، في فرنسا منذ

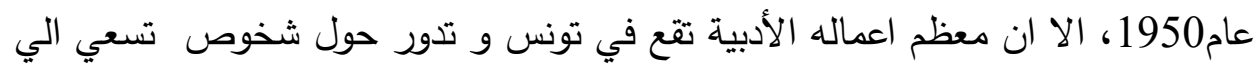

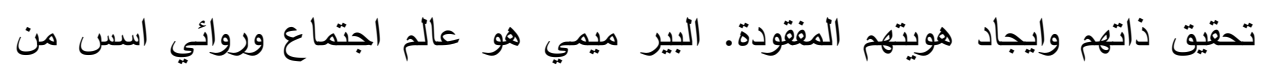
خلال رواياته الادب التونسي و المجربي كما انه يعتبر من احد رموز الكفاح ضد هدماند

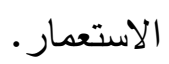

تمثل رواياته "تمثال من ملح" و "الفرعون" اشكالية البحث عن الذات، فمن خلال تلك

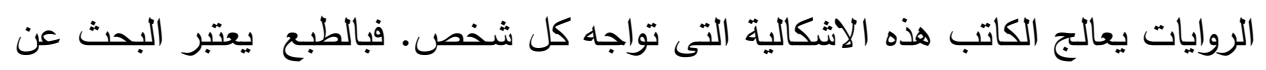

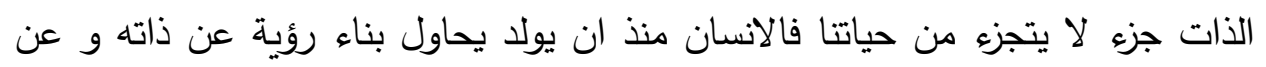

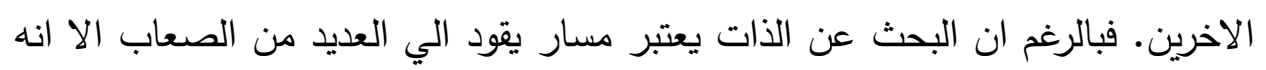
يبقي مثل الضوء الصاطع في سماء حياتتا. وفي هذا البحث سنقوم بدراسة الاسباب التي دفعت الثخوص الي الانغماس في مشروع البحث عن الذات مثل الشعور بالتمزق والثعور بالوحدة و الشعور بسلب الحقوق. وسنوضح كيف سعت الثخوص الي تحقيق ذاتهم من خلال العديد من المظاهرواخيرا سوف نتتاول مصير هؤلاء الثخوص الباحثين عن ذاتهر.

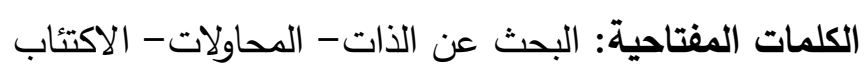


Quête de soi et dépression chez Albert Memmi

\section{Quête de soi et dépression chez Albert Memmi}

\section{Chahinaz Sabri Mohamed}

(Assistante au département de français- Faculté de pédagogie)

»L'assimilation abandonnée, la libération du colonisé doit s'effectuer par la reconquête de soi «....

Bien entendu, dans un roman, les personnages sont un des piliers fondamentaux sur lequel l'histoire est construite et organisée : ils subissent les événements qui ravitaillent la trame et l'intrigue du roman. Nous pouvons ainsi dire que « toute histoire est histoire des personnages ». En effet, notre romancier nous présente des actants qui ont mené une vie extrêmement semblable à la sienne. Ainsi, nous pouvons dire que l'aspect autobiographique dans ses romans est assez flagrant. Mais, notre auteur ne manque pas de donner une touche fictionnelle à ses récits. Il fait alors osciller le lecteur entre le réel et le fictif : "C'est une écriture libre qui mêle le rêve et la réalité «.

Dans cette étude, nous nous proposons d'étudier la quête de soi chez Albert Memmi dans «La Statue de Sel » et «Le Pharaon». En effet, Les œuvres de Memmi sont peuplées de protagonistes colonisés qui aspirent reconquérir leur soi perdu pour jouir de l'émancipation de toutes les contraintes qui leur sont imposées et pour aboutir à se libérer de tous les sentiments macabres dans lesquels ils se sentent engouffrés. L'être memmien veut en finir avec l'effondrement de son moi ; c'est pourquoi, il s'engage dans une quête qui vise la récupération et la réalisation de soi, et surtout la réconciliation avec soi-même et avec autrui. Au demeurant, la quête de soi aide l'homme à dévoiler les secrets de son âme, à savoir les buts de ses actes et à réaliser ses désirs refoulés et ses rêves souhaités. A travers ses romans, l'auteur en question illustre le désir intense de ses personnages de se reconnaître voire de 
trouver un sens à leur vie à travers cette quête. De fait, l'expérience de la récupération de l'identité est considérée comme un voyage dans lequel le soi se trouve au bout d'une voie dans une soif de quête et d'enquête. Ce qui nous mène à l'examen de plusieurs interrogations : Quels sont les sentiments déclencheurs et catalyseurs de cette quête ? Quels sont les aspects de cette recherche de soi ? Ces aspects sont-ils positifs ou négatifs ?Comment cette quête s'est-elle terminée c'est à dire les personnages ont-ils réussi à trouver leur soi perdu ? Nous tenterons de répondre à ces questions à travers le schéma quinaire proposé par Greimas et Larivaille:

Etat initial $\rightarrow$ Complication ou Force Perturbatrice $\rightarrow$ Dynamique $\rightarrow$ Résolution ou Force équilibrante $\rightarrow$ État final

Il nous incombe de montrer que chaque étape de ce schéma constitue une transformation d'un état à un autre par " un élément qui enclenche le procès de transformation de la dynamique qui l'effectue(ou non) et d'un autre élément qui clôt le procès de transformation.»( ) Ce schéma nous aide dans l'étude des rôles accomplis par les personnages des romans en question ainsi qu'il montre l'évolution et l'enchaînement des cinq séquences menant de l'état initial vers l'état final.

État initial:

C'est l'étape de la présentation des éléments nécessaires pour la compréhension du récit comme le lieu, l'époque et les personnages. Ainsi, dans « La Statue de Sel », l'auteur introduit son récit par un personnage-enfant qui vit en totale harmonie au sein de sa famille dans une modeste maison : «Le monde de mon enfance fut rassuré, protégé par ce souffle d'asthmatique qui dissipait les frayeurs de mes réveils solitaires. »(SDS, p.17). Il sent la sécurité auprès de son père au point qu'il le considère comme une divinité protectrice de ce monde "Effrayé, je me serrais contre mon père dans le grand lit familial, lui mettant les jambes sur le ventre. »(SDS,p17). De même, il nous parle de sa mère qui avait l'habitude de se réveiller tôt afin

$$
\text { العدد الرابع والعشرون (الجزء الثالث) } 2018
$$

مجلة كلية التربية- جامعة عين شمس 19 
de préparer le café et parfumait la chambre par cette odeur : «Mes matins d'espoir doivent embaumer le café maure. "(SDS, p18). Ainsi, le personnage évoque l'intimité bienheureuse de cet espace, « une chambre au fond de l'impasse Tarfoune ", qui malgré sa modestie, lui procure la sérénité.

Quant au « Pharaon », ce roman débute par l'introduction d'un personnage qui vit avec sa femme dans une villa. Il avait l'habitude de voyager à Paris où il travaillait et où il a fait la connaissance de sa femme Allegra. Il est à la fois archéologue et professeur. Au début, tout semblait aller bien. Sa vie était idéale : il était « un bon époux » et un « bon père ».Il savait très bien s'occuper de ses recherches qu'il publiait régulièrement. Il acceptait sa religion juive : « je suis à la fois un époux, un père, un fils et un ami, un archéologue et un professeur, je suis d'ici et de Paris et, en un sens également Jérusalem que je n'ai jamais vue.»(Le Ph,p58). Nous remarquons la différence qui existe entre les deux personnages, dans les romans en question, dans leur âge, leur statut et leur niveau social et économique. Cependant les deux protagonistes jouissent d'une vie stable.

Complication :

C'est l'étape dans laquelle l'on se heurte à la perturbation de la situation initiale. Ainsi, cette étape signale la transformation de l'état équilibré de la vie de deux personnages. Dans « La Statue de Sel », nous remarquons la rupture de la vie harmonieuse de l'enfant après avoir quitté l'impasse et après s'être installé au Passage : " il n'a pas duré longtemps ce bonheur confortable. » (SDS,p33) . Il est à signaler que le nom de ce «Passage » symbolise la transition d'une phase de vie stable en une autre agitée. Ce changement ne se limite pas strictement à un cadre spatial mais plutôt à une modification qui ébranle le déroulement du cours de la vie du personnage .

العدد الرابع والعشرون (الجزء الثالث) 2018

()

مجلة كلية التربية- جامعة عين شمس20 
Dans le même élan, la crise identitaire de Gozlan, le héros dans «Le Pharaon », apparaît dès qu'il a décidé de s'installer définitivement dans sa ville natale après être devenu un expert dans le domaine de l'archéologie. Il découvre sa perte anthologique et son importunité parmi les autres et va même jusqu'à ignorer son propre pays. Ainsi, les personnages vont souffrir de maladies psychologiques compliquées. Nous allons nous contenter d'analyser le portrait psychologique du personnage principal dans les romans en question. Pour ce faire, nous allons nous référer au schéma suivant cristallisant les sentiments morbides qu'éprouve l'être memmien :

En effet, Memmi peint ses personnages comme des êtres fragmentés et fracassés. Ainsi, la fracture intérieure du personnage avec son soi prédomine dans «La Statue de Sel », et le mène à glisser dans un abîme insurmontable et percutant. Il se sent menacé d'un danger qui réside souvent dans le fait de se sentir tirailler entre l'Orient et l'Occident .

Un des symptômes de ce déchirement entre les deux différents univers apparaît lorsque le petit Alexandre, le personnage principal de notre roman, n'arrive pas à comprendre son maître qui parlait souvent en français : «cette langue qui était la plus importante dans l'enseignement». Ce « bilinguisme colonial» l'engouffre de plus en plus dans cet éclatement intérieur. Le héros décrit le véritable traumatisme qu'il affronte dès sa plus tendre enfance, à l'âge de sept ans en arborant : " j'étais devant un gouffre..... le maître ne parlait que français, je ne parlais que patois comment pourrions-nous jamais nous rencontrer. » (SDS,pp.43,44).

Parallèlement, nous remarquons cet écartèlement entre l'Orient et l'occident dans "Le Pharaon » à travers les paroles de Paolo adressées à son père : «Tu sympathises bien avec les Arabes. La

$$
2018 \text { العدد الرابع والعشرون (الجزء الثالث) }
$$

مجلة كلية التربية- جامعة عين شمس 21 
France, c'est l'Europe, l'Europe, c'est la civilisation, l'avenir.(....) Bon tu as choisi les Arabes, c'est ton affaire, moi, j'ai choisi les Français.... »(Le Ph,p.63). Ainsi, nous remarquons comment l'être memmien se trouve dans l'obligation de choisir entre deux mondes antagoniques : l'Orient, sa terre natale et l'Occident, porteur d'un bel avenir. Nous pouvons alors nous référer dans ce stade d'analyse aux propos d'Amin Maalouf : «il (l'Occident) demeure porteur des valeurs les plus avancées de notre temps, alors ceux qui s'attaquent à lui sont souvent porteurs de régression «.

Il est à souligner que ce décalage entre l'Orient et l'Occident s'étend aux autres romans de notre écrivain. Par exemple, dans «Agar », le narrateur décrit l'histoire d'un jeune médecin juif tunisien qui s'est marié avec une femme française. Il montre l'échec de cette relation confrontée à la différence de culture.

L'écrivain même montre ce combat entre ces deux univers contradictoires dans La terre intérieure en déclarant : "J'étais alors en plein tumulte intellectuel et affectif, entre Orient et Occident. » Notons que bien que notre auteur fut condamné à vivre ce divorce culturel, il a réussi à maîtriser la langue française, celle du colonisateur : "Albert Memmi s'affirme avec vigueur comme un maître de l'écriture d'une grande rigueur alors que le français n'est pas sa langue maternelle «.

Devons-nous dans ce stade d'analyse signaler que ce combat entre l'Orient et l'Occident est repris dans les œuvres de Mohamed Dib, écrivain algérien qui a tenté à travers ses romans de « communiquer au lecteur le drame de la déchirure provoquée par la rencontre conflictuelle des cultures dans le contexte colonial.» De même, nous remarquons que ces deux écrivains magrébins ont choisi de s'exprimer en langue française car elle est devenue « paradoxalement un instrument de libération servant à déconstruire l'image fabriquée par le colonisateur «. 
D'autre part, Alexandre dans « La Statue de Sel » se sentait condamné à vivre ses fragmentations depuis sa naissance car ses parents tunisiens lui ont choisi le nom d'Alexandre " en hommage à l'Occident prestigieux. " (SDS,p.107).Il est à noter que le nom, en général, a pour rôle de nous singulariser, nous protéger de l'anonymat. Il est considéré comme un moyen conventionnel permettant de s'identifier et de communiquer. Au demeurant, Il fonde et établit l'identité parce qu'il affirme la personnalité de l'être, le distingue de l'autre et lui réclame une certaine particularité. Cependant, dans le roman en question le nom a une influence négative sur l'état psychique du protagoniste. Le nom se soustrait à sa valeur et à sa fonction et ne sert qu'à accentuer son déchirement : Le seul énoncé de mon nom me faisait honte ......... je détestais mon prénom de toutes mes forces. »(SDS, p107).Ce prénom était pour notre personnage un facteur de honte parce qu'il révèle les différentes identités qu'Alexandre porte en lui :Alexandre représente l'Occident, Benillouche fait allusion aux origines africaines indignes et Mordekhai fait référence à sa judéité. Ainsi, notre personnage se trouve fragmenté entres ces trois identités incompatibles. Cette multitude d'identité, au lieu d'être une source de richesse assurant la personnalité et réalisant un bon atout pour être ouvert à l'Autre, elle l'a mené à un déchirement sans issue . Pourtant, le choix de cet onomastique est expressif puisque le nom " désigne les personnages, les inscrit dans l'univers social et le système des oppositions du roman, condense des informations et symbolise les acteurs ». Le prénom " Alexandre' désigne celui qui écarte et « repousse le guerrier (ennemi) » qui est, dans notre cas, le colonisateur. De plus, le choix de ce prénom précisément n'est pas par hasard ; nous pensons que les parents d'Alexandre ont choisi ce prénom en espérant que leur fils soit à l'image du fameux conquérant Alexandre le Grand. Celui-ci a fondé un énorme empire qui s'étendait jusqu'à l'Inde. Au surplus, le père et la mère du 
nouveau- né ont peut-être voulu qu'il fût comme Alexandre Jannée qui est un grand prêtre juif et un successeur d'Aristote ou comme d'autres personnes célèbres portant ce prénom.

En outre, le scindement de soi de notre protagoniste est toujours accru par le complexe d'infériorité : il ne cesse de se décrire comme «pauvre ».Cet adjectif a une connotation péjorative qui touche le sens propre du mot. Il est à noter qu'Alexandre a pu poursuivre ses études grâce à une bourse obtenue par le lycée. Ses collègues voient qu'il n'est pas à la hauteur des lyciens. Ainsi, ils se moquent de lui et le traitent d'un air hautain : " ils se regardaient en ricanant, ils se répétaient " langage de concierge. »(SDS,p.126)

Un autre élément engouffre le personnage de plus en plus dans ce déchirement viscéral, c'est sa langue natale. En effet, la langue maternelle de l'être joue un rôle essentiel dans son identification. Elle ne représente pas seulement un moyen qui lui permet de communiquer avec autrui, mais aussi elle exerce aussi une influence indéniable dans la construction de son identité .Pourtant, dans « La Statue de Sel ", nous remarquons que la langue s'évade de sa substance et de son rôle et ne fait qu'augmenter l'éclatement de soi chez notre personnage :" Lorsqu' 'il m'arrive de me parler en patois j'ai toujours l'impression bizarre ..... d'entendre une partie obscure de moi-même. "(SDS,p.314)

En outre, Gozlan dans, "Le Pharaon », était convaincu que la langue arabe, au moment de la colonisation, était stérile : l'épreuve s'incarne surtout lorsqu'il ne s'oppose pas à l'opinion de Bouzid. Celui-ci qualifie la langue maternelle « d'infirmité »(Ph,p.57). Gozlan sait que la langue française lui sert dans son démarche politique et combative contre la colonisation.

Il est à remarquer que le sentiment de la fragmentation de soi est accru chez Gozlan à cause de sa religion : "On ne connaît de nous, et nous-mêmes ne connaissons de nous-mêmes, que l'image de serfs misérables pour survivre..... »(Le Ph, p.144). L'emploi du 
Chahinaz Sabri Mohamed

terme « misérable »montre la vie lamentable que le juif est condamné à vivre. Gozlan, en tant que juif, sent qu'il appartient à un groupe minoritaire, mis en accusation et agressé. Il est à noter qu'Albert Memmi, lui-même, a déclaré sa fragmentation avec sa religion : "Ce qui me frappe d'abord lorsque je me considère comme juif, c'est que je n'aime pas ainsi me considérer «.

En fait, après avoir montré comment les personnages souffrent d'une fragmentation intérieure avec eux- mêmes, nous allons nous permettre de passer à la fragmentation des personnages avec autrui. La question qui s'impose est donc la suivante : est-ce qu'il y a une relation entre la distanciation des êtres avec eux-mêmes et leur désunion avec leur entourage ?

La réponse est certainement oui car l'homme, qui souffre d'un soi fragmenté et tronqué, est toujours en conflit avec les autres puisque «L'autre est un doublet du soi ».De même, le personnage, qui éprouve la séparation avec les autres, n'arrive pas à coïncider avec soi-même. Axel Kahn a affirmé que l'autre est « la condition de soi(...)plus encore une partie de son être «.

Ainsi, dans « La Statue de sel », nous remarquons que la tendance psychique du personnage est « «la dé-mixtion, la déliaison » même avec ses parents. Bien que la famille soit un des piliers exerçant une influence positive sur l'état psychique de l'être humain, ceci n'est pas le cas dans ce roman. Notre romancier s'acharne à nous montrer la honte que Alexandre a éprouvé pour la première fois envers ses parents : « je les vis, pour la première fois, gauches et honteux d'eux-mêmes(...).Ils chuchotaient probablement gênés de leur patois, qui m'apparut vulgaire et déplacé. »(SDS,p. 59). Ainsi, on voit naître un gouffre entre Alexandre et ses parents après s'être inscrit à l'école française, lieu fréquenté par la classe bourgeoise. Dès cet instant, il prend conscience de la pauvreté de

العدد الرابع والعشرون (الجزء الثالث) 2018

()

مجلة كلية التربية- جامعة عين شمس25 
son père et l'analphabétisme de sa mère et il éprouve de la honte envers eux .

De même, rejeté par autrui, le Moi chez le héros memmien se trouve disloqué puisque chaque homme cherche à trouver son Moi à travers les jugements et la vision de l'Autre. « .... Autrui apparaît comme nécessaire à la constitution de ce moi ». Dans «La statue de sel », lorsque les camarades d'Alexandre se moquaient de lui à cause de son prénom, il devenait antagoniste envers soi-même ainsi qu'envers ses camarades : « je détestais mon prénom de toutes mes forces et aussi mescamarades. » (SDS,p.106) Une brutale dissociation naît ainsi entre le héros et son entourage. Il se trouve souvent agressé par « la foule nerveuse » qui « se moquait $»$ de lui ; ce qui lui rend la vie de plus en plus difficle et pesante . Également, dans «Le pharaon », Memmi nous montre le décalage qui existe entre Gozlan et l'autre. Ce dernier n'accepte pas la religion juive. A titre d'exemple lorsque l'équipe du journal « Patriote » accuse Alexandre d'être sioniste, Gozlan le défend : " Benillouche n'est pas plus sioniste que la plupart des juifs. »(Le Ph,p. 44) Selon Gozlan, Alexandre souhaite seulement trouver une solution nationale pour le peuple. Nous remarquons qu'il y a un lien qui rattache « Le Pharaon » à « La statue de Sel », c'est la reprise du personnage principal de «La Statue de Sel » qui se trouve toujours accusé d'être juif. Ainsi, Gozlan sent toujours l'irritation et la fragmentation à cause des gens antisémites qui hantent son pays : « Il aurait fallu une explication fort longue, et périlleuse pour ses relations avec les jeunes gens qui auraient fini par s'irriter à leur tour. »(Le Ph,p. 58)

Nous devons signaler que malgré le désaccord que Gozlan éprouve envers cette équipe de journal, il a accepté de faire partie du journal et de rédiger les pages culturelles. Il a aussi bâti une relation professionnelle agréable avec les autres journalistes. 
En outre, les fêtes et les cérémonies hebdomadaires que Gozlan fréquente augmentent son sentiment de fragmentation : dans les conversations et les débats entre les gens ayant de religions hétérogènes, chacun éprouve le seul droit d'accaparer le pays et d'imposer sa propre religion. C'est pour cette raison que Gozlan avait l'habitude de ne pas participer à ses discussions : « il n'avait nulle envie d'ajouter son grain de sel. »(Le Ph,p.44)

De même, ce malaise social se manifeste aussi à travers sa relation avec sa femme Allegra : «(...) entre Allegra et Armand il y a ce désert, qui depuis des années gagne sans cesse(...). Leur vie commune est depuis longtemps un roman mort où rien ne se passe plus. »(Le Ph,p.151). Armand souffre d'un terrible problème de communication avec son épouse car ils ne sont pas sur la même longueur d'onde. De plus, le personnage masculin entretient un monologue intérieur où il exprime son sentiment d'être scindé avec son fils : «Quel toupet ! Il n'a pas cessé de m'attaquer, je me suis à peine défendu pour ne pas le blesser et en somme il me reproche. »(Le Ph,p.64).Ces propos miroitent qu'il était souvent en désaccord avec son cadre familial. Cette fragmentation atteint son apogée quand il éprouve la rupture même avec Dieu : Armand ne « croyait ni à Dieu ni à sa grâce. »(Le Ph,p.52)

Puisque le personnage memmien éprouve souvent une distanciation avec soi-même et avec son entourage, il devient une proie facile à la méconnaissance de soi et d'autrui. Commençons par traiter la méconnaissance de soi ou la dépersonnalisation dont souffre l'être memmien:

En effet, d'après le dictionnaire de la psychologie, l'étrangéisation de soi ou la dépersonnalisation est définie comme " un état où l'individu ne se reconnaît pas lui-même comme personne" . Cet état, selon Sartre, surgit lorsque l'être n'arrive pas à être en symbiose avec son entourage car l'individu se connait à travers l'autre. L'homme ne peut acquérir une image fidèle de soi que

$$
\text { العدد الرابع والعشرون (الجزء الثالث) } 2018
$$

مجلة كلية التربية- جامعة عين شمس27 
grâce au regard d'autrui. Ce dernier est considéré comme un miroir dans lequel on est reflété.

Ayant des relations fragmentées avec les autres, Alexandre se trouve noyé dans le sentiment de la méconnaissance de soi. On constate que ce sentiment, qui immerge son âme, est accompagné d'une crise identitaire. Ceci est très remarquable lorsqu'il se pose souvent la question suivante : « qui-suis-je enfin ? »(SDS,p.109).Ainsi nous pouvons dévoiler que le quêteur memmien tente de rechercher en lui-même qui il est et ce qu'il représente pour les autres. C'est le « qui suis-je » pour soi bien sûr, mais c'est encore qui « suis-je » pour autrui.

Également dans « Le Pharaon », l'écrivain nous a montré qu'Armand s'était ignoré lui- même et il se demande « qui il était » $(\mathrm{Ph}, \mathrm{p} .17)$. Cette question revient dans les œuvres en question comme " un besoin constant de justification, d'explication, de plaidoyer » de la part de l'auteur qui se trouve en proie à ce sentiment de l'étrangéisation de soi et qui se pose cette « question lancinante et sans fin(..) Qui suis-je exactement « .

D'autre part, nous pouvons aussi signaler que la domination du régime colonial sur la terre natale du personnage memmien a creusé à l'intérieur de son soi cette dépersonnalisation. C'est dans les propos d'Alexandre, héros de La Statue de Sel que surgit en filigrane cette impression : « je me découvris irréductiblement étranger dans ma ville natale » (SDS,p.110). Ainsi, le terme « étranger » montre comment le personnage souffre de la méconnaissance de son soi sous la colonisation. Le protagoniste sent qu'il vit dans une atmosphère culturelle différente à la sienne. Par conséquent, il sent qu'il est étranger même au sein de son pays . De même, la pauvreté de notre protagoniste déculpe ce sentiment : lorsqu'il rejoint le lycée, il fréquente une autre couche sociale, la 
classe bourgeoise. Ainsi, il éprouve qu'il est étranger parmi cette classe riche.

Signalons aussi que la religion juive des personnages dans les œuvres en question ne fait qu'augmenter ce sentiment. A titre d'exemple dans Le Pharaon, notre héros, juif dans un pays à grande majorité musulmane, se heurte au gouffre existant entre les deux religions. De même, Alexandre dans « La Statue de Sel » affirme : " L'antisémitisme était une caractéristique des autres »(SDS,p.275). Memmi fait allusion au mouvement antisémite qui « est apparu sous une forme virulente à l'époque contemporaine, plus précisément au XIX siècle. Ce mouvement a mené à des persécutions variables envers les juifs . Nous pouvons, de même, nous référer aux propos de Simone de Beauvoir qui a montré la répugnance et la désapprobation des Juifs de la part des antistémistes : «Les juifs sont des autres pour l'antisémite « .

En outre, il paraît que le sentiment d'étrangéisation de soi n'épargne aucun être memmien .Ceci est marqué lorsque l'auteur dans Le scorpion affirme : «Vous est-il arrivé, en vous rasant, d'avoir une impression bizarre, de vous poser une question devant votre propre visage dans le miroir (...) Vous découvriez un étranger(.....). » Le mot " étranger » prend plusieurs sens. Il signifie parfois le fait d'être d'un autre lieu ou appartenir à une autre culture ou comme, d'après Baudelaire, l'étranger c'est un homme énigmatique. Le héros memmien sent toujours qu'il est une personne énigmatique qui ne se reconnaît pas lui-même. Albert Memmi n'est pas l'unique écrivain qui a montré comment l'homme dominé souffre de ce sentiment. La plupart des écrivains maghrébins ont également cristallisé la domination de cette sensation morbide sur le colonisé. A titre d'exemple, nous pouvons nous référer aux propos d'Amin Maalouf lorsqu'il a déclaré : « Le blessure intime (...) est d'abord lié à ce sentiment, acquis depuis 
l'enfance, d'être irrémédiablement minoritaire, irrémédiablement étranger où que je sois «.

Ainsi, le colonisé se trouve tourmenté par cette impression et il va même jusqu'à éprouver un certain déracinement intérieur : "L'exil est toujours difficile à assumer qu'il soit de l'intérieur et de l'extérieur ».Le déracinement d'après le dictionnaire Le Robert, signifie « l'arrachement » ou «l'exil ». Le protagoniste memmien avait l'impression qu'il était arraché de ses racines ou de sa terre natale. Il est en exil non parce qu'il a une vocation d'exilé mais qu'il mène une existence inadéquate à sa propre ville natale.

Après avoir étudié les causes de la dépersonnalisation des personnages, nous allons maintenant tenter d'aborder le sentiment de la méconnaissance des autres que l'être memmien éprouve. Ayant rompu avec sa mère, Alexandre la considère toujours comme « une étrangère » dont il avait toujours peur : «j'avais peur de ma mère, ma mère me devenait opaque(...) Elle m'était étrangère (....) » (SDS,p.181). Il nous incombe de montrer que le rapport fils-mère est de caractère choquant et morbide. Dès qu'Alexandre a fréquenté l'école, il atteint un niveau culturel plus élevé de celui de sa mère. C'est pour cette raison qu'il sent que sa mère se trouve dans un monde totalement différent du sien . Nous pouvons dire aussi que sa mère était toujours entièrement occupée de la ménagère et elle n'avait pas assez de temps pour se rapprocher de son fils et savoir ses besoins, ses sentiments, ses secrets et ses rêves surtout lorsqu'elle a donné naissance à six autres enfants. Alors ils demeurent étrangers l'un par rapport à l'autre. Ceci est marqué par cette phrase très significative et très émouvante proférée par le héros : « De ma mère, j'évitais de parler, je n'en pouvais rien dire. $»($ SDS,p.212)

Remarquons comment ce sentiment hante Alexandre au point de se sentir souvent cerné par des étrangers : « Autour des sauvages étrangers la rue demeurait déserte et silencieuse. »(SDS,p.37). Le

$$
\text { العدد الابع والعشرون (الجزء الثالث) } 2018
$$

مجلة كلية التربية- جامعة عين شمس30 
Chahinaz Sabri Mohamed

mot "étranger" renvoie non seulement à son entourage qu'il considère souvent comme des sauvages et des fantômes, mais aussi à sa situation en tant que colonisé. Il faut noter que l'emploi des deux adjectifs " déserte" et " silencieuse " est en parfait accord avec l'état d'âme du protagoniste. Ce qui reflète que celui-ci éprouve souvent que sa terre natale est devenue extrêmement aride et austère bien qu'elle soit peuplée de gens.

Parallèlement, Armand Gozlan dans Le pharaon, se plaint toujours d'être entouré de gens qu'il ignore. Il demande toujours « qui était les siens»(Le Ph,p.17). Le terme « siens »dans cette citation fait allusion au peuple qui l'accuse d'être juif. Quoiqu'il essaye d'entretenir des relations agréables avec les autres, ils lui sont toujours étrangers. Ce sentiment est né à cause du refus d'autrui . Ainsi, cette citation exprime le désir de connaître l'autre afin de pouvoir se reconnaître.

Comme nous l'avons déjà signalé, il existe un mur psychique entre les êtres memmiens et leur entourage. Ce mur les sépare des autres, par conséquent ils se trouvent condamnés à la solitude affreuse et étouffante. Pourtant, l'individu est un être social par sa nature. L'homme ne peut être humain qu'à travers son intégration et son insertion avec l'altérité : "Chaque homme a besoin de ses semblables pour percevoir le monde extérieur dans sa totalité ». Il doit vivre « en commun ». C'est ainsi que l'autre représente un autre moi, sans l'autre, le moi devient traqué, amputé, incomplet voire altéré .

Cependant, Memmi nous présente toujours des personnages qui éprouvent la solitude. Il est à noter que la solitude, qu'elle soit voulue ou imposée, est toujours source d'inquiétude et de détresse. Dans La statue de sel, nous constatons que les paroles du héros montrent qu'il est toujours « seul ». Il est à noter qu'Alexandre a éprouvé ce sentiment très tôt pendant son enfance. La première fois qu' il a éprouvé cette émotion, il était installé avec ses camardes

2018 العدد الرابع والعشرون (الجزء الثالث)

()

مجلة كلية التزبية- جامعة عين شمس31 
dans une colonie de vacances. Il était exaspéré et accablé par la solitude et l'isolation : « je me trouvais, pour la première fois, jeté seul dans le monde. »(SDS,p.63)

Parmi les symptômes de ce sentiment, nous pouvons signaler la souffrance et la douleur morale dans lesquelles Alexandre est plongé : « Je pleurais à grosses larmes avec des hoquets de poitrine....... »(SDS,p.64).Par cette description tordante, le narrateur nous dissèque l'âme ébranlée du personnage. Celui-ci, noyé dans les larmes du chagrin, se lamente sur son existence: «Lorsque les voix se trouvaient assourdies, encore plus seul, je pleurais sur ma solitude. »(SDS,p.64)

Selon Alexandre, cette solitude est toujours labyrinthique puisqu'il ne trouve jamais d'issue. Ayant souvent le sentiment suraigu de la solitude qui « pinça le cœur »(SDS,p.59),le personnage est confiné dans une « complète solitude »(SDS,p.241)même au lycée. Ainsi, il se sent enfermé psychologiquement dans un huis clos. Voilà pourquoi, nous pouvons dire que la cause de cette émotion réside dans la désertification relationnelle et l'absence «d'interlocuteurs valables $\ll$.

De même, dans Le pharaon, l'être memmien éprouve un état d'exclusion et de solitude. Gozlan sent qu'il est définitivement « seul, comme il l'avait toujours été depuis tant d'années........ »(Le Ph,p.13).Il éprouve ce sentiment de solitaire dans le monde depuis des années. Il est à signaler que ce sentiment qui envahit Armand est causé par l'absence perpétuelle de son entourage familial : " mes enfants n'y venaient plus que rarement et Allégra ne rentrait guère avant huit heures .»(Le Ph,p.26).Son fils n'était pas si attaché à lui, bien que ce dernier lui vouait un amour très vif. Paolo avait sa vie politique et il participait à des opérations punitives et à des attentats et il n'acceptait pas son père "sympathisant avec les arabes »(Le Ph,p.63). Quant à sa fille qu'il aime et considère

العدد الرابع والعشرون (الجزء الثالث) 2018

()

مجلة كلية التربية- جامعة عين شمس32 
comme « le fruit de ses entrailles »(Le Ph,p.28), elle était occupée par une relation amoureuse avec Jean Pic qu'elle a connu dans la galerie Lumbroso.

Il est à noter que ce n'est pas seulement Gozlan qui se trouve torturé par la solitude, mais aussi presque tous les personnages memmiens goûtent continuellement à ce sentiment amer. La bienaimée de Gozlan a affirmé qu'elle a éprouvé cet affreux sentiment. Elle restait « seule des journées entières »(Le Ph,p.133) ; elle n'a jamais été « aussi solitaire que dans cette ville surpeuplée »(Le Ph,p.135). Remarquons le contraste entre « solitaire » et « surpeuplée »qui met l'accent sur l'état d'exclusion complète du personnage bien qu'il soit entouré de gens. Il est séquestré et engouffré dans ce sentiment. Ceci est marqué par la répétition de l'adjectif « seul »dès le début du roman.

Également, Alexandre éprouve un exil psychologique même quand il est entouré de gens. Il met en évidence son propre état par une phrase très significative : «je retrouvai l'affreuse solitude dans la foule indifférente. »(SDS,p.67). L'emploi de l'adjectif « affreuse » élucide par excellence son état d'âme. Il sent qu'il est claustré dans sa solitude même au sein de sa famille. Memmi montre d'une part, l'inaptitude de son personnage à s'intégrer avec son entourage et d'autre part il nous cristallise à quel point notre protagoniste est refusé par les autres. Ainsi, le héros sent qu'il est importun et parasite aux yeux des autres aussi bien qu'aux yeux de sa famille. Voilà pourquoi, il passe la plupart de son temps avec un livre dans sa maison qu'il considère comme une cellule de prison: « (...) j'étais seul avec mon livre.... »(SDS,p.128).

Il est à noter que l'emploi récurrent du terme " seul » est assez expressif. Il nous donne l'impression que le héros est privé d'amour et que sa vie est vide de toute tendresse et d'amitié. Mais comme l'homme ne peut s'identifier que par rapport aux autres, le personnage memmien n'arrive presque jamais à trouver son soi tout

العدد الرابع والعشرون (الجزء الثالث) 2018

()

مجلة كلية التزبية- جامعة عين شمس33 
en restant seul. C'est pour cette raison que Heidegger a employé l'expression « l'être au monde « .

En effet, les personnages de Memmi rappellent ceux de Michel Tournier souffrant souvent de la solitude et de la perte de leur identité : " Tous les romans de Michel Tournier sont des romans de la solitude, qui racontent la perte de l'autre et la découverte de la faille intime, et ses héros sont tous de grands solitaires (....), mais fascinées également par l'unité, tentées et rebutées par l'altérité, recherchant inlassablement la présence et le contact d'autrui . « .....

Cependant, devons-nous signaler que la solitude était parfois pour l'être memmien un refuge, un moyen de protéger son Moi en décomposition. Dans « La Statue de Sel » la rupture d'Alexandre avec les autres le pousse à « s'isoler du monde. »(SDS,p.204). Nous avons l'impression qu'il tente de construire des barricades qui le protègent d'autrui. Il passait des jours sans parler à son clan. Il se laissait séquestrer dans son mutisme. Ce comportement déculpe de plus en plus sa solitude car « être seul n'est pas une manière d'être sans l'autre, mais une altération de mon rapport à l'autre ». Alexandre essaie de se convaincre qu'il n'a pas besoin de l'autre. Il tente de fourrer dans son esprit l'idée que lorsqu'il vit seul et isolé, il va vivre dans la satisfaction : "Vivant seul, je vivais en paix. »(SDS,p.77)

Il faut mentionner qu'Alexandre a eu recours à un processus psychologique accompli par le Moi nommé : la rationalisation. Cette dernière est définie comme un « un procédé par lequel le sujet cherche à donner une explication cohérente logique, acceptable, morale à une attitude, un sentiment dont il ne perçoit pas les véritables motifs ". C'est un des mécanismes de défense dont « se sert le moi dans les conflits susceptibles d'aboutir à une névrose » . Mais évidemment le soi ne peut être replié des autres sois. 
L'insertion et la communication entre les individus sont les composantes fondamentales de la santé psychique.

De même, Gozlan avait l'habitude de s'isoler afin de se consacrer à ses études et à ses recherches. Celui-ci «sourit de cette autosatisfaction vaniteuse, vite dépiste : solitude orgueilleuse mais peuplée (.....) »(Le Ph,p.13) .

Il paraît que Gozlan tente de créer des prétextes d'isolement pour cacher la vraie raison de sa solitude volontaire : « la peur des autres »: la peur d'être choqué ou blessé par l'autre. Il préfère rester solitaire et décide de s'isoler croyant que l'autre avec qui il peut partager la confiance et l'amour, va lui causer la souffrance. Le voici dire ces propos dans un monologue intérieur lorsqu'il a senti la souffrance de sa fille : « ..... Pourquoi souffre-t-on le plus cruellement par ce que l'on aime le plus ? Pourquoi la souffrance accompagne-t-elle l'amour comme l'ombre la lumière ? »(Le Ph,p.28)

Soulignons- nous qu'il y a souvent un rapport entre la solitude et le vide. Ainsi, nous nous souscrivons à l'opinion de Cioran qui a affirmé que : « quand, par appétit de solitude, nous avons brisé nos liens, le vide nous saisit : plus rien, plus personne ».Memmi tâche de nous démontrer le vide existentiel intrinsèque à sa condition humaine en plongeant ses personnages dans le néant. Au fil de la lecture du roman, on constate que ce sentiment accablant ronge le protagoniste: « j'étais prisonnier du vide. »(SDS,p77). Ceci est marqué aussi par l'emploi fréquent du mot « désert . «

De même, dans « Le Pharaon », Gozlan sent qu'il vit « seul dans un monde vide »(Le Ph,p.349). L'actant est immergé et englouti par un monde vide de tout amour et de tout bonheur.

Un dernier détail compliquant la vie des personnages memmiens est la privation des droits. Le colonisé, dans son pays « n’a ni droits, ni devoirs : il ne possède rien.... ». Niant et ignorant les droits nécessaires de mener une existence digne de respect, le colonialisme 
Quête de soi et dépression chez Albert Memmi

s'acharne à tuer moralement à petit feu les compatriotes maghrébins. Memmi nous montre fortement la déchéance de l'humanité pendant la période de la domination. Le colonisé vit dans un état de "sous humanité ". L’être memmien se trouve plongé dans la misère irréparable et la pauvreté la plus extrême. Le narrateur considère la colonisation comme un arbre reproduisant des fruits qui ont un goût amer et âcre. Les héros, dès leur apparition dans les romans, sont privés et dépossédés de leurs droits. Le colonialisme empêche d'établir la démocratie et la liberté. Il est évident que le régime colonial suit un système injuste. Il fournit plusieurs avantages et bénéfices au colon et traite le colonisé comme un esclave dépourvu de ses moindres droits.

Ainsi, dans « La statue de sel », Alexandre affirme : « je suis un batard de ma ville natale. »(SDS,p.110). Le statut du peuple colonisé ne lui inspire que la honte de lui-même et il se considère comme un batard dépossédé de ses droits. Il éprouve le sentiment étouffant d'être exploité et terrorisé par la puissance encombrante du colonisateur. Alexandre se voit donc indigne de mener une vie respectable dans sa terre natale : "Toujours je me retrouverai Alexandre Mordekhaï, Alexandre Benillouche, indigne dans un pays de colonisation $\gg($ SDS,p.109). Cette indignation est marquée par cette affirmation : « je suis de statut indigne .»(SDS,p.108)

De même, Alexandre voulait rompre avec la pauvreté qui empêchait la réalisation de ses aspirations. Cette famille misérable ne possède pas les droits et les biens essentiels qui garantissent sa survie. Il habite avec toute sa famille dans une petite chambre très modeste se trouvant dans un quartier très pauvre. Il a déclaré qu'il a passé des jours étourdi par la faim au point de se sentir noyé dans « un univers de misères. "(SDS,p.175).Ainsi, l'argent était le catalyseur des sentiments amers dont souffrait le personnage. Il était un facteur de honte et d'envie. Il voudrait rompre avec la pauvreté et gagner beaucoup d'argent afin d'accéder à la gloire qu'il aspirait

العدد الرابع والعشرون (الجزء الثالث) 2018

()

مجلة كلية التربية- جامعة عين شمس36 
Chahinaz Sabri Mohamed

conquérir Mais, nous remarquons qu' à la fin du roman, il est convaincu que l'argent ne fait jamais le bonheur : " Je me fiche de l'argent. »(SDS,p.252)

Notons que le thème de l'aliénation est fondamental dans le deuxième roman de notre corpus «Le Pharaon ». Le héros a du mal à reconquérir ses droits. Il est toujours dépossédé de ses droits et de ses biens, traité comme un « citoyen de deuxième catégorie »(Le Ph,p.162).C'est pourquoi, il aspire à trouver une issue pour rompre cette émotion angoissante et mettre fin à sa misère. Il paraît que le héros demeure perpétuellement infligé dans cette déperdition des droits .

Il est à signaler que Memmi présente des personnages non seulement aliénés mais aussi plongés dans la violence dont les manifestations sont très flagrantes : " ...les maisons détruites, les femmes violées, les provisions souillées..... »(Le Ph,p.176). Il met l'accent sur les événements sombres qui s'insèrent dans la tourmente destructrice de la période coloniale, exposant ainsi les personnages à «l'extrême violence »(Le Ph,p.206). Gozlan sent qu'il n'est pas sécurisé sur sa terre natale : «Oui, je suis de ce pays mais, je n'y suis jamais rassuré...... » (Le Ph,p.278).Au fil de ces événements ténébreux, les rêves et les désirs de Gozlan sont de plus en plus frustrés. Nous trouvons souvent la ségrégation et le racisme qui règnent comme un souverain absolu sous ce régime exploiteur. Dynamique:

Cette étape montre les moyens et les démarches utilisés par les personnages afin de résoudre la perturbation. Ainsi, ces sentiments ont été les éléments nodaux qui ont incité les personnages à suivre cette quête. Ils tenteront de récupérer et de réaliser leur soi : " Tous les héros tentent de définir leur identité et de résoudre leurs contradictions à partir d'un moi déchiré entre plusieurs ressentis comme incompatibles ». Pour ce faire, ils ont eu recours à l'amour,

العدد الرابع والعشرون (الجزء الثالث) 2018

()

مجلة كلية التربية- جامعة عين شمس37 
à l'engagement et au travail. Commençons par traiter le premier point qui est : l'amour.

Déçus par les relations interpersonnelles traumatisantes et boiteuses, les quêteurs memmiens tentent de trouver dans le lien amoureux une sorte de restauration de leur moi : «Dans l'amour l'homme mutilé cherche à reconstituer son intégrité première ». Au départ, dans « La statue de sel », notre écrivain met en vive lumière le rôle majeur de l'amante d'Alexandre dans le fait d'atténuer la souffrance de son bien-aimé. Sa relation avec Ginou , cette fille tunisienne, l'éloigne de sentir ses souffrances et ses maux : «Mais enfin, je goûtais au bonheur. » (SDS,p.196)

Il est à souligner que Ginou était pour Alexandre une sorte d'échappatoire pour fuir une existence inacceptable. Sa relation amoureuse avec elle lui donne l'espoir de goûter aux charmes et aux délices de la vie : " Mon aventure avec Ginou me découvrit que, si j'étais né dans une impossible situation historique, la vie pouvait avoir un goût de miel .»(SDS,p.196) .Notre auteur démontre par excellence comment Alexandre trouve auprès de sa bien-aimée la chaleur et l'affection qui lui manquent dans cet univers glacial.

C'est pour cette raison qu'elle représente dorénavant, pour lui, l'être le plus précieux au monde : «En deux jours, sans que je lui eusse parlé davantage, elle me devint la plus chère au monde. » (SDS,p.198)

Il paraît qu'Alexandre crée avec sa bien aimée un monde particulier où il se sent plus libre. Il a la vive impression qu'il vit dans un monde utopique dépourvu d'amertume et de restriction. $\mathrm{Sa}$ relation avec elle étanche la soif de trouver son soi et d'assouvir les désirs inhibés dans son inconscient. Très tôt, elle est devient son idole. Nous remarquons qu'il se refugie en elle et lui parle de ses désirs et de ses rêves. Après être tombé amoureux de Ginou, Alexandre aspire à un avenir optimiste et prometteur. Il est persuadé que cette relation amoureuse aurait un rôle incontestable dans sa

2018 العدد الرابع والعشرون (الجزء الثالث)

()

مجلة كلية التربية- جامعة عين شمس38 
Chahinaz Sabri Mohamed

formation identitaire : « .....je parlai à Ginou de ce qui me tenait à cœur : de moi-même, de mes ambitions, de mes certitudes. $\gg($ SDS,p.199)

En effet, Alexandre est fasciné par sa bien aimée au point qu'il rêve d'elle tous les soirs au début de leur relation : " je rêvais d'elle, tous les soirs, tous les soirs en m'endormant, je répétais son nom, Ginou, Ginou »(SDS,p.200). Selon Freud, le rêve est« la réalisation hallucinatoire de désir " . Alexandre rêve de ce qu'il désire réaliser dans la réalité. Il souhaite continuer sa vie auprès de sa bien aimée .

Parallèlement dans « Le Pharaon », le recours à l'amour est une tentative faite par Gozlan pour se récupérer et donner un sens à sa vie : L'amour procure à l'homme la satisfaction de se sentir indispensable dans ce monde ». Il éprouve le besoin de trouver une personne qui lui fait confiance et qui l'écoute afin de combler le manque et la solitude qui l'entourent. C'est l'amputation de toute proximité et de toute harmonie dans la vie conjugale de Gozlan qui le pousse à se jeter dans les bras d'une jeune fille algérienne appelée Carlotta. Ainsi, cette femme devienne une conquête à laquelle il n'a pu résister et il a perdu toutes ses forces et ses armes. Il la considère comme étant l'étoile qui illumine son existence. Sa bien-aimée lui procure souvent un rayon d'espérance : "Carlotta ma chance, la dernière, Carlotta ma fête, mon étoile qui danse sur la ville .» (Le Ph,p.109)

Il est important de signaler que cet amour a donné un sens à son existence, puisque Carlotta lui a assuré les besoins affectifs aspirés. Grâce à cette union amoureuse, il retrouve même sa jeunesse bafouée: "Grâce à Carlotta, je me suis arrêté de vieillir, j'ai rajeuni. »(Le Ph,p.109). Cette relation le revêt de jubilation : « je ris de bon cœur, de cœur bon, avec la poitrine qui se gonfle....... je suis heureux »(Le Ph,p.152). C'est pour cette raison qu'il s'enfonce de plus en plus dans cette union. Il éprouve une passion puissante

$$
\text { العدد الرابع والعشرون (الجزء الثالث) } 2018
$$

مجلة كلية التربية- جامعة عين شمس39 
envers son amante qui s'arrose chaque jour davantage. Nous constatons à quel point cet amour a transformé l'angoisse du personnage en sérénité. Ce rapport a soulagé la tristesse qui a serré son âme et son esprit pendant des années. Il avait l'impression qu'il était enterré dans un lieu sinistre et que c'est Carlotta qui lui a offert l'occasion de s'en sortir pour vivre en plein bonheur : « .... Je me suis bâti un tombeau et lorsque tu es apparue, tu m'as proposé d'en sortir, de vivre avec toi au grand jour »(Le Ph,p.345). Elle lui a donné l'occasion d'éprouver tous les sentiments espérés.

Il est à signaler que Carlotta éprouve aussi envers Gozlan une grande affection : " tu es l'homme dont je souhaite être la femme »(Le Ph,p.139). Gozlan 1'a inondée par son affection et par sa sollicitude. C'est grâce à lui qu'elle a retrouvé son soi : «Tu m'as rendue à moi-même » (Le Ph,p.139).Ainsi nous remarquons l'harmonie qui couvre les deux conjoints.

Enfin, nous pouvons déclarer que le personnage memmien a pensé que l'amour est le chemin idéal menant vers l'identification du soi. Mais à la fin du roman, Gozlan abandonne Carlotta et s'installe à Paris avec sa femme et ses enfants. Quant à Alexandre, l'amour n'était qu'un étape dans sa vie. Même Ginou l'a quitté quand il a renoncé à son rêve d'être un médecin et il a choisi d'être un professeur de philosophie

Passons maintenant à la deuxième tentative à laquelle les personnages ont eu recours dans ce projet identitaire : ils éprouvent le besoin de s'engager afin de récupérer leur soi perdu .

L'engagement dans les romans en question né d'un besoin d'agir face aux événements iniques qui se reproduisent au sein de la terre natale. Nous référant au dictionnaire, nous trouvons que l'engagement est un « acte ou attitude de l'intellectuel de l'artiste qui, prenant conscience de son appartenance à la société et au 
Chahinaz Sabri Mohamed

monde de son temps, renonce à une position de simple spectateur et met sa pensée ou son art au service d'un cause « .

Ainsi, Memmi présente des êtres qui s'engagent au service de leur pays pour reconquérir leur liberté et celle d'autrui. Remarquons que notre auteur a donné une importance indéniable à l'engagement. Il a même mentionné dans son essai «Portrait du colonisé » que ce dernier a le devoir de s'engager pour accéder à la liberté. De même, il insiste sur le fait que tout le peuple doit s'unir afin de réaliser les bénéfices de la solidarité. Autrement dit, selon lui , pour être un homme, il faut être responsable. L'être trouve un sens à sa vie à travers le soulèvement et l'action. Ainsi, nous pouvons dire que l'auteur adopte « la littérature d'affirmation de soi et de contestation $\ll$.

D'ailleurs, il nous paraît indispensable de cristalliser que Memmi était influencé par les pensées et les écrits de Sartre en ce qui concerne le refus de la colonisation et la nécessité de l'engagement. Ce dernier représente un acte "d'auto-défense » contre l'oppression du colonisateur, cet être ignoble et sans âme. Nous pouvons même ajouter que les germes de la résistance bouillonnent dans le cœur de l'homme dominé comme un désir sauvage. C'est ce que Sartre a déclaré dans la préface de Portrait du colonisé : «Maintenus par un système oppressif au niveau de la bête, on ne leur donne aucun droit, pas même celui de vivre(...) quand un peuple n'a d'autre ressource que de choisir son genre de mort(..) C'est son malheur qui deviendra son courage ; cet éternel refus que la colonisation lui oppose, il en fera le refus absolu de la colonisation $\ll$.

Ainsi, selon l'optique memmien et sartrien, c'est le régime colonial qui incite le colonisé à s'engager. L'engagement est pour le colonisé une sorte de révolte contre son existence amère. Celui-ci essaye de se révolter afin de se libérer de l'oppression, du déracinement voire de la surexploitation. Ainsi, les personnages à

العدد الرابع والعشرون (الجزء الثالث) 2018

()

مجلة كلية التربية- جامعة عين شمس41 
travers leur engagement expriment leur exaspération face aux événements qui se déroulent sur leur territoire .

A titre d'exemple, dans "Le Pharaon », notre écrivain a réussi par excellence à montrer comment le système colonial despotique a incité Gozlan à entamer cette procédure éthique. Ce sont les perturbations qui se trouvent en Tunisie qui le poussent à prendre au sérieux sa tâche. Il sent qu'il se trouve devant une obligation morale à laquelle il ne peut se soustraire. L'être memmien n'est donc pas passif et ne reste plus les bras croisés. Ainsi, dans le roman en question, le protagoniste lutte contre une situation inacceptable en défendant les droits des oppressés : «......il s'était engagé par fidélité aux idéaux appris à l'Université, par solidarité avec tous les opprimés. »(Le Ph,p.66)

En outre, Gozlan a senti la nécessité de son adhésion à la politique afin de reconquérir son identité et celle d'autrui. Nous remarquons que « l'engagement politique de Gozlan »(Le Ph,p.295) n'est pas seulement pour un but individuel, mais il est aussi au nom de la collectivité. Il a ainsi déployé tous ses efforts afin d' " être un homme de quelque part, un homme parmi les hommes ». Le personnage choisit ainsi de suivre un chemin positif : Gozlan avait l'habitude d'assister une fois par mois au salon de Mme Teman afin de discuter sur la situation économique et politique du pays avec les autres membres. Ce salon « figurait un prototype, soigneusement reproduit par toute la bourgeoisie locale.. »(Le Ph,p.86).

Également dans «La statue de sel », l'engagement est né de la noirceur des événements qui accompagnent souvent le personnage comme une malédiction. A la déclaration de la guerre, Alexandre trouve le besoin de s'engager avec ses compatriotes afin de « les aider à retrouver, à garder leur dignité. »(SDS, p.307). Nous tenons à montrer qu'Alexandre pendant la période de la guerre s'allie avec son peuple pour sauver son territoire .

العدد الرابع والعشرون (الجزء الثالث) 2018

()

مجلة كلية التربية- جامعة عين شثس42 
Il paraît que la guerre a appris au protagoniste la solidarité. Il va participer volontairement à la vie communautaire. Nous remarquons comment les pulsions personelles sont transformées en pulsions sociales : « je ne me sentais plus le droit de me soustraire individuellement à la catastrophe. »(SDS.p.304). Il est clair qu'Alexandre voit que l'engagement est un acte sain, un chemin qui le mène à la réalisation de son soi. Il éprouve le besoin perpétuel de s'engager avec l'autre pour retrouver son identité. Se référant à Sartre, il affirme que « le surgissement d'autrui affecte profondément le sens de ce que je suis ». Ainsi, notre protagoniste n'accepte pas de demeurer paralysé devant cette crise : "Que faire, mon Dieu, que faire ! Pour tous ces hommes qui souffraient et mouraient dans les camps... » (SDS,p.188).À travers ce monologue, nous constatons comment Alexandre désire rendre service à son peuple juif opprimé. Il sent l'obligation de mettre fin à cette situation qui devenait de plus en plus catastrophique. C'est pourquoi, il a décidé de s'installer dans un camp afin d' " aider les autres à vivre. »(SDS,p.305)

Nous remarquons que la sauvegarde de la liberté de son pays était son premier souci au point que sa séparation avec Ginou n'a pas violemment touché son cœur bien qu'elle fût la seule femme aimée. Nous pouvons conclure que son engagement est plus fort que son amour ; ce qui affirme les sentiments affectifs qui lient Alexandre à son territoire. Cet amour de la femme se transforme en amour pour la patrie .

De même, notre romancier cristallise l'enrôlement du protagoniste dans "Le Pharaon " lorsqu'il a assisté à " une importante réunion politique »(Le $\mathrm{Ph}, \mathrm{p} .58)$ où il a rencontré un homme « qui allait jouer un grand rôle dans la crise tunisienne :Louis Perillier »(p68), un politicien français qui avait pour but « d'amener la Tunisie vers l'indépendance. »(p..69) Notre héros aspire à l'amélioration de la situation de son territoire. Il essaye de

العدد الرابع والعشرون (الجزء الثالث) 2018

()

تجلة كلية التزبية- جامعة عين شمس43 
briser les barricades qui empêchent l'accession à l'émancipation Nous constatons que Gozlan défend les valeurs de sa patrie. Il avait l'ambition de résister en combattrant toutes les sortes de l'aliénation et de la dépossession afin de récupérer son pays violé . C'est pour cela qu'Habib Bourguiba, lors de sa rencontre avec Gozlan, «remercia l'archéologue pour ce qu'il avait fait pour le peuple tunisien. $\gg($ p.113)

Nous pouvons ainsi dévoiler que le patriotisme est pour Gozlan le fil d'Ariane qui le mène vers la réalisation de son soi. Nous nous référons aussi aux propos de Corneille : « vis... pour servir encore ton pays.... vis pour servir l'État ».Ces mots, si précieux reflètent l'exaltation du patriotisme. C'est une passion raisonnée et instinctive qui lie l'homme à son territoire comme l'affirme Sartre dans la préface de l'essai intitulé « Portrait du colonisé » en insistant sur le fait que c'est le système colonial qui attise l'amour de la patrie au cœur du colonisé .

Nous pouvons ainsi déclarer que la notion de la patrie, dans les romans en question, a souvent une dimension émotive et affective : «L'œuvre de Memmi se caractérise par(...) une passion très forte éprouvée pour le pays natal ou la communauté d'origine qui n'exclut nullement la critique voire le sarcasme " . Notre écrivain cristallise les sentiments d'amour et d'attachement que Gozlan éprouve à l'égard de son pays à travers ce monologue intérieur : " Pourquoi ne quittons -nous pas ce minuscule pays ? Ici ma peinture et ma vie. $\gg($ Le Ph, p.122)

Nous remarquons aussi « l'engagement journalistique » (p.91) de Gozlan : il dirige les pages culturelles du journal «Patriote ». Il nous convient de montrer que Gozlan trouve un grand plaisir dans l'écriture. Il espère libérer sa plume. Il avait même l'habitude chaque soir de rédiger dans ses carnets «les événements de la journée pour en tirer la leçon. »(p.31)

العدد الرابع والعشرون (الجزء الثالث) 2018 
Également, Alexandre dans « La Statue de Sel », avait l'habitude d'écrire son journal intime. L'écriture était pour lui un refuge de sa vie amère, une évasion de sa situation économique et de ses conditions familiales. L'écriture était un moyen qui lui permttait d'accéder à une autre vie plus adéquate à son niveau intellectuel : " Pour m'alléger du poids du monde, je le mis sur le papier : je commençai à écrire. Je découvris l'extraordinaire jouissance de maîtriser toute existence en la recréant .» (SDS,p.123)

En outre, nous constatons que l'identification de soi des personnages est liée au devoir du travail. Ce dernier demeure une des manifestations de cette quête. Nous trouvons que la plupart des psychanalystes ont donné une grande importance au travail. A titre d'exemple Freud voit que le travail joue un rôle efficace dans la maintenance de l'équilibre de la vie de l'être. Dans les romans en question, le travail assouvit le désir impérieux des protagonistes de s'affirmer. De même, il satisfait leurs besoins intellectuels et matériels.

Le travail chez Gozlan, l'archéologue, avait un aspect efficace et avantageux. C'était, pour lui, un moyen pertinent pour réaliser ses ambitions. Notre personnage voudrait laisser une empreinte dans le domaine de l'archéologie : « (.....) il avait toujours travaillé dur, pour mériter les renouvellements de ses bourses, pour satisfaire et honorer ses bienfaiteurs de la communauté, pour réussir son concours d'entrée à l'école, pour obtenir sa chair, pour.....pour vivre .»(Le ph,p.63) .Nous pouvons ainsi mentionner que le travail était, pour Gozlan, un substitut du bonheur ou plutôt l'essence de sa vie : " son travail était plus important pour lui que tout au monde »(p.263). Il était souvent occupé par son enseignement et par ses recherches qu'il publiait souvent. Puis, il a travaillé dans une boutique de cadeaux afin de consacrer son temps pour ses candidats de doctorat et pour son projet qu'il espère réaliser mais malheureusement faute de temps ce projet n'a pas vu le jour :

العدد الرابع والعشرون (الجزء الثالث) 2018

()

مجلة كلية التربية- جامعة عين شمس45 
Gozlan « imagina de constituer une Bible des temps modernes, à l'instar de l'autre, mais issue de l'humanité entière, où il inclurait les textes les plus représentatifs de tous les peuples, de sorte que le lecteur disposerait de la quintessence de la sagesse humaine. »(Le ph, p.14).

De même, il est à mentionner qu'Alexandre était prêt à déployer un grand effort afin de maîtriser et prononcer la langue française comme ses camarades et de fortifier ses points faibles. Il prend au sérieux cette tâche : «Bien sûr, j'ai essayé, plus ou moins consciemment d'imiter mes camarades..........je me suis obligé à écouter des opéras, à suivre des pièces de théâtre, notant soigneusement la biographie des auteurs et les renseignements sur les œuvres. »(SDS,p.122)

Il nous convient de montrer que le travail sérieux pendant les années scolaires est pour notre héros une manière de se rapporter à soimême. Alexandre a la certitude que le travail est un moyen qui lui permet de s'affirmer. C'est pour cette raison que nous remarquons son enthousiasme accroît jour après jour : " j'avais violemment envie de manifester, d'affirmer ce que j'étais. »(p.125).Assoiffé de s'identifier, Alexandre ne se limite pas aux informations qu'il apprend au lycée, mais il tente de s'enrichir en découvrant des connaissances supplémentaires avec beaucoup d'enthousiasme et d'ardeur pour se réaliser : " je ne me contentais pas de nos connaissances scolaires toutes digérées .»(p.124).Ainsi, il prend l'habitude de lire à tout moment pour assouvir pleinement son appétit pour la connaissance : «......je lisais à table, dans la rue en attendant la sonnerie, au lit jusqu'à une heure du matin .»(p 124). Nous pouvons aussi souligner qu'Alexandre, à travers la lecture, retrouve ses ambitions, ses désirs voire son avenir.

Il est important de signaler que notre personnage était motivé pendant les années scolaires par deux professeurs : Marrou, son professeur de français et Poinsot, son professeur de philosophie. Le 
premier menait une existence semblable à notre héros : «Dans mes visites à Marrou, je l'écoutais parler, parce qu'il parlait à la fois de lui et de moi. »(p.242). Alexandre était ébloui par lui car, c'était un homme très cultivé malgré sa pauvreté. De même, il a réussi à maîtriser une langue qui n'est pas la sienne. Quant à Poinsoit, il était son « idéal humain »(p.242).C'est grâce à lui qu'il voudrait devenir professeur de philosophie. Il avait l'habitude de l'accompagner après l'école et lui racontait ses « derniers événements intérieurs .»(p.243).

D'ailleurs, Memmi s'acharne à nous montrer la puissance et l'insistance de son personnage à travailler même dans des conditions difficiles. Celui-ci voudrait atteindre ses fins malgré les difficultés et les obstacles qu'il affronte. Nous constatons sa volonté de s'affirmer et d'être un homme brillant : "Je supportais de travailler dans la chambre où jouaient, criaient, se disputaient six enfants.... »(p.137).

En outre, il est important de montrer que le protagoniste a aimé travailler pendant son adolescence devant un miroir ;ce qui reflète l'envie d'Alexandre de regarder souvent son image. Le recours à la glace était une tentative de retrouver son soi. Alexandre tente de se reconnaître à travers ce miroir : " J'aimais assez me regarder, longuement, interrogeant le miroir sur ce que j'étais....... $»($ p.138) .Il est à signaler que le miroir n'est pas seulement un matériel d'accessoire qui permet à l'homme de voir son visage, mais aussi il donne à l'homme l'occasion de fonder une vision à laquelle le Moi aura la capacité de se reconnaître. Cette vision permet à l'homme de s'identifier et de pénétrer les tréfonds de son âme. Ainsi, nous pouvons signaler que cet objet optique est un « instrument de l'Illumination », un espace de connaissance voire de reconnaissance du monde où nous vivons. L'emploi du verbe " interroger » prouve que le protagoniste attend une réponse du miroir concernant son existence .

العدد الرابع والعشرون (الجزء الثالث) 2018

()

مجلة كلية التربية- جامعة عين شمس47 
Nous pouvons dans ce stade d'analyse se référer aux propos de Taher Ben Jelloun «Avec le miroir, tu verras ton visage et ta pensée. Tu verras ce que les autres ne voient pas quand ils te regardent. C'est un miroir pour les profondeurs de l'âme, pour le visible et l'invisible « .

Résolution

Cette étape marque la fin des péripéties. Ainsi, on se pose la question suivante : est-ce que cette quête a permis aux personnages de s'enfuir de leur crise identitaire?

La réponse à cette question est négative puisqu'ils se sont heurtés à la dépression. Cette dernière peut être définie comme « un ténébreux état d'âme, un vécu de tonalité triste dont l'ampleur et l'intensité varient comme la durée « .

Les héros ont alors suivi un chemin qui ne conduise qu'à la mélancolie et à la dépression. Dans « Le Pharaon », Carlotta a demandé à Gozlan de mettre fin à leur relation :elle s'ennuie de passer le long de sa vie à attendre quelques moments volés avec Gozlan. Elle veut sortir du tombeau dans lequel elle vit avec son amant et elle veut vivre avec lui au grand jour : «Je te veux tout entier...je veux des enfants de toi...je veux tous les instants de ta vie. Je veux tout ou rien. »(Le Ph, p.347)

Cette tentative faite par Carlotta était dans le but de renouveler la flamme de l'amour avec Gozlan et de le pousser à quitter sa femme. Pourtant, Gozlan a choisi de quitter Carlotta et de continuer sa vie avec sa femme : " Allegra fait partie de ma coquille(...) sans Allegra, trop vieux prisonnier libéré, je me heurtais, me blesserais au monde, je serais amnésique et nu.» (Le Ph,p.333)

Mais comme Gozlan n'arrive pas à accepter l'idée de la séparation définitive de sa bien-aimée, il sombre dans des crises de dépression et tombe dans les zones les plus ténébreuses d'un amour éphémère qui ne lui procure que le malheur. L'auteur s'acharne à nous élucider l'état psychique de Gozlan : celui-ci est hanté par ses

العدد الرابع والعشرون (الجزء الثالث) 2018

()

مجلة كلية التربية- جامعة عين شثس48 
Chahinaz Sabri Mohamed

souvenirs avec Carlotta :....Il passait son temps en promenades solitaires dans tous ces endroits où il avait été si heureux... »(Le Ph,p.361). Ses souvenirs ineffaçables nourrissent sa peine. Même ses enfants ont remarqué pour la première fois « le désarroi »(Le Ph,p.361) de leur père : son fils commente l'état lamentable de son père en proférant ces propos : « Pauvre papa.........il est tout désorienté, il ne sait comment se défendre. »(Le Ph,p.361)

En outre, à la suite de la déclaration de la nouvelle constitution tunisienne, Gozlan désespère. Réclamée comme « un pays arabe et de religion musulmane »(Le Ph,p.357), la Tunisie n'est plus laïque aux yeux du protagoniste. Pendant l'ère coloniale, Gozlan a souffert amèrement de sa condition juive et après la décolonisation, il aspirait à la libéralisation. Mais malheureusement, il découvre qu'il n'y trouve pas sa place. Sa condition juive l'a enfermé dans un enfer éternel duquel il n'arrive pas à s'en sortir. Son état, en tant que Juif, est considéré comme une accusation, un problème qui entrave la réalisation de son soi et de ses rêves assoiffés.

Obsédé par l'idée qu'il devienne de plus en plus opprimé dans son pays, le personnage pense qu'il est condamné à vivre aliéné, marginalisé et exclu même après la libération de son pays. Notons d'ailleurs qu'après l'indépendance de la Tunisie, la souffrance et le malaise du peuple s'accentuent car le système opprimant a totalement exploité les fortunes du pays. L'occupant ne part qu'après avoir ravagé le pays. Nous pouvons aussi nous référer aux propos de notre romancier : «C'est triste à dire, mais le sort du colonisé d'hier s'est encore dégradé après le départ de celui qu'il considérait comme son oppresseur. L'indépendance n'a pas rendu le décolonisé heureux.......La décolonisation était indispensable, mais elle a été un fruit amer teinté de deuils «.

Dans le même élan, toutes les tentatives du personnage de La Statue de Sel étaient dans l'espoir de changer la situation de son

$$
\text { العدد الرابع والعشرون (الجزء الثالث) } 2018
$$

بجلة كلية التربية- جامعة عين شمس49 
pays, mais, malheureusement cette dernière est devenue de plus en plus catastrophique. Memmi s'acharne à nous montrer comment Alexandre vivait au-dessus d'un volcan de guerre qui ne s'éteignait pas. " Nous eûmes nos victimes, exécuté par punitions, erreurs ou plaisanteries, nos femmes violées, nos demeures pillées »(SDS,p.265). Ces événements accentuent certainement la tristesse et l'angoisse du protagoniste qui affirme que : "L'angoisse des premiers jours nous reprit, mais une angoisse rageuse, agitée, de bêtes traquées. »(SDS,p.303).

Nous remarquons que les événements funèbres du pays ont détruit son rêve d'être professeur de philosophie : «Mais c'est bien la fin, je ne serai pas professeur »(SDS,p.366).Alexandre avait la ferme certitude qu'il tourne dans une spirale éternelle ou un cercle vicieux et qu'il est condamné à vivre ces situations à l'infini : « je n'aurai jamais la solution de mon problème car je suis problème. »(SDS,p.346). Le personnage se rend compte qu'il n'arrivera jamais à mener une vie humaine dans son propre pays puisque ce dernier est impuissant face à la guerre et au régime colonial. La dépression atteint son paroxysme au point que le héros se sent prêt à mourir : « vivant.... Je sens que je suis mort.»(SDS,p.350). Il ne s'agit pas de la mort charnelle mais plutôt de la mort psychique. Le colonialisme dominateur et autoritaire lui a fait perdre son soi, son propre être au point qu'il se sent comme un mort vivant.

De même, ce qui rend la situation de plus en plus sordide c'est qu'Alexandre est atteint de tuberculose. Force de signaler que notre romancier a fait exprès de choisir cette maladie honteuse qui est souvent « liée à la pauvreté, à la dégradation des conditions économiques $\gg 1$. Le personnage sent ainsi qu'il est tombé dans l'abîme de la séquestration morale incarnée dans la solitude et la séquestration physique causée par sa maladie incurable.

Memmi cristallise l'égarement de notre personnage à travers ce fragment : «trois....cinq....six.....huit.....dix, onze. Onze ou 
douze ? Comment savoir ? Le monde est mort et je suis sans montre. » (SDS,p.376).Alexandre se sent déboussolé et désorienté au point qu'il a perdu même la notion du temps. Nous constatons comment il s'engouffre dans l'abîme de la souffrance à cause des circonstances si cruelles et déshumaines qui se passent dans son pays natal. Il est enfermé dans l'ombre de la tristesse au point qu'il considère que le monde entier est mort. Cet état dépressif le conduit à songer au suicide, l'unique moyen qui lui permette de s'évader de son existence avilissante et outrageante : « J'ai pensé à mourir, à quitter le monde entier .»(SDS,p.368)

État final

Cette étape marque le dénouement du récit, le retour des personnages à une situation stable, différente de la situation initiale. Ainsi, Etant complètement déçu, ayant perdu tout espoir en la réalisation de ses rêves, Alexandre décide de s'enfuir vers l'Argentine « pour cesser de se retourner sur lui-même, afin de ne pas être changé, comme la femme de Loth, en statue de sel ». Il est à noter que Memmi clôture son roman par un fragment assez symbolique: " Comme je me trouvais mal à l'aise à regarder la mer violette, qui me faisait des grâces de sorcière, se creusant puis se haussant, me tendant les bras, je descendis dormir dans la cale. »(SDS,p.377).Notons que le violet est une couleur qui marque la douceur mais en même temps qui donne l'impression de la solitude ou de la détresse. C'est la couleur de « la passion et l'intelligence, l'amour et la sagesse », mais aussi celle du « deuil ou du demideuil «.

De même, la mer a une double connotation : elle est à la fois le symbole de la douceur et celui de la méchanceté. Considérée comme une mère qui tend les bras à son enfant, elle est une figure allégorique de la maternité comme le précise Bachelard : "L'eau nous porte. L'eau nous berce. L'eau nous endort. L'eau nous rend notre mère » Cependant, elle représente une image passive, celle de 
l'adversaire, lorsqu'elle incarne la tristesse. C'est ainsi que la mer peut être « une ennemie qui cherche à vaincre et qu'il faut vaincre » ; « l'image de la vie et celle de la mort «.

Ainsi nous pouvons démystifier que la mer symbolise la terre de naissance puisque toutes les deux reflètent le retour à la source première. Elle est le symbole du pays natal de notre protagoniste, sa patrie qui lui procure le bonheur et lui offre la tendresse ; mais en même temps elle engendre le chagrin et le rend mal à l'aise. Nous avons l'impression que le protagoniste, face à cette mer, contemple l'infini et la dépression .

Quant à Gozlan, il finit par être convaincu que Carlotta ne mérite pas qu'il bouleverse sa vie pour s'enfermer à nouveau dans une nouvelle prison. Le voici dire : "Toute vie commune est un enfer à domicile ; au mieux un purgatoire qui ne débouche jamais sur le paradis »(Le Ph,p.347). Ainsi, il prend la ferme décision de quitter son pays afin de fuir et d'oublier ses souvenirs avec sa bienaimée : "Nous ne pouvons que gérer nos faiblesses ; il emmena donc Allegra et les siens à Paris. »(Le Ph,p.362) .Gozlan a donc choisi de prendre avec lui sa famille, car il est persuadé que s'il reste seul, il se transformera en un être faible : " il savait qu'il ne pouvait pas vivre seul. »(Le Ph,p.363) .

De même, nous pouvons dévoiler que ce départ est pour lui la résolution la plus pertinente après que la Tunisie est devenue une république dont l'Islam est la religion et l'Arabe est la langue officielle. Le protagoniste s'enfuit de son pays espérant un futur épanouissant loin de la corruption, du chaos voire de la misère assaillant son pays après la décolonisation : «..... Dans ce temps nouveau si ardemment souhaité, conquis parfois au prix de terribles épreuves, règnent encore la misère et la corruption, la violence sinon le chaos $\ll$.

Force de mentionner qu'après la décolonisation, il se fit « un grand mouvement d'exode des cadres et des intellectuels »(Le 
Chahinaz Sabri Mohamed

$\mathrm{Ph}$, p.362) qui ont été déçu par la condition déplorable de leur terre natale. Comblés de dépression, ils« vont chercher ailleurs l'emploi de leur talent et leur savoir « .

Ainsi, le départ de Gozlan vers Paris a une bonne influence sur lui . Il aspire à respirer en plein poumons l'air de l'indépendance : " .......il était bien exilé dans son pays natal ; la preuve en était qu'il respirait mieux à Paris qu'à Tunis. »(Le $\mathrm{Ph}, \mathrm{p} .372)$. Il réussit à occuper des postes prépondérantes après quelques mois de son installation à Paris. Cependant, il n'arrive pas à oublier son pays natal qui représente tout son patrimoine culturel et son bagage de souvenirs d'enfance : " il espérait retourner un jour dans son pays natal. »(Le Ph,p370). Memmi nous montre à la fin comment Gozlan se trouve envahi par des sentiments nostalgiques envers son pays : «Et si, malgré tout, il est envahi par quelque nostalgie, il va dîner en célibataire dans le quartier de Belleville ou celui de Montmartre, où se sont installés de nombreux restaurants tunisiens. Rien ne console autant que les nourritures de l'enfance »1. (Le Ph,p.376)

De tout ce qui précède, nous pouvons résumer le rôle des personnages selon le schéma actanciel proposé par Greimas:

Rôles /

Romans Destinateur Destinataire Sujet Objet AdjuvantOppossant La Statue de Sel Alexandre Alexandre Alexandre La quête de soi Ginou Marrou Poinsoit Le régime colonial, sa famille La classe bourgeoise Le Pharaon

\section{Gozlan Gozlan et son peuple}

Gozlan L'affirmation de soi Carlotta Le régime colonial, sa famille

D'après ce schéma, nous pouvons distinguer dans les romans en question six catégories d'actants participants : Le Sujet et l'Objet sur l'axe du désir ou du vouloir : les personnages memmiens s'engagent dans une quête qui vise la quête de leur identité. L'Adjuvant et l'Opposant se situent sur l'axe du pouvoir : dans les

العدد الرابع والعشرون (الجزء الثالث) 2018

()

مجلة كلية التربية- جامعة عين شمس 
romans, objets de notre étude, nous trouvons des personnages qui aident les êtres memmiens dans leur projet identitaire tels Ginou, Marrou et Poinsoit dans « La Statue de Sel » et Carlotta, la bienaimée de Gozlan dans « Le Pharaon ». Alors que le système colonial, ce régime autoritaire, ne fait que décupler la crise identitaire des protagonistes .De même, la famille de ces derniers était un pilier qui influe négativement sur leur état psychique. Les gens qui ont des religions hétérogènes à celle de nos personnages avaient aussi une influence déplorable sur l'être memmien. En plus, la classe bourgeoise dans « La Statue de sel » augmente la fragmentation identitaire du personnage. Le Destinateur et le Destinataire sont unis sur l'axe du savoir ou de la communication : comme le personnage memmien décide de reconquérir son soi, il accomplit alors plusieurs rôles conjointement : il est à la fois le destinateur, le destinataire et le sujet.

En somme, nous avons montré que les personnages ont subi la fragmentation psychosomatique. Au demeurant, nous avons étalé que Memmi a ingénieusement présenté des personnages affligés par la solitude morale et psychique. De même, nous avons souligné comment le personnage se sent comme un inconnu dans un univers qui lui est à la fois étrange et étranger. Nous avons signalé aussi que les personnages ont été éreintés par la perte de leurs droits. Ensuite, nous avons étudié les manifestations de la quête et les tentatives faites par les personnages pour la reconquête de leur soi et à la fin de cette étude, nous avons cristallisé comment les protagonistes n'ont pas réussi à s'identifier et ils ont pris la décision de quitter leur pays natal. 


\section{Orientation bibliographique:}

1(Corpus (par ordre chronologique):

-MEMMI, Albert, La Statue de Sel, Paris, Gallimard,1966

Le Pharaon, Paris, Julliard, 1988

2 (OEuvres d'Albert Memmi (par ordre chronologique)

-MEMMI, Albert, Portrait du juif, Paris, Gallimard,1962

-MEMMI, Albert, Le scorpion, Paris, Gallimard,1969

-MEMMI, Albert, La terre intérieure, Paris, Gallimard,1976

-MEMMI, Albert, Portrait du colonisé, Paris, Gallimard,1985

-MEMMI, Albert, Portrait du décolonisé, Paris, Gallimard,2004

3 (Autres oeuvres (par ordre alphabétique):

-BEN-JELLOUN, Taher, L"enfant de sable, Paris, Seuil, 1987

-CORNEILLE, Pierre , Horace, Paris, L.gf,1986

-GOETHE, Johann Wolfgang, La souffrance du jeune Wrerther, Paris, Livre de Poche, 1999

-SARTRE, Jean Paul, Les mouches, Paris, Gallimard,1947

-DE BEAVOIR, Simone, Le deuxième sexe, Paris, Gallimard, 1976

-TOURNIER, Michel, Les Météores, Paris, Gallimard,2007

4(Ouvrages et études de critique sur Memmi:

-DEJEUX, Jean, La littérature Maghrébine d"expression française, Paris, Presses universitaires de France, 1992

-BONN, Charles \& GARNIER, Xavier, Littérature francophone, Paris, Hatier, 1997

-JOUBERT, Jean Louis, Littératures francophone du monde arabe, Paris, Nathan,1994

-NDIAYE, Christiane, Introduction aux littératures francophones, Canada, Les presses de l"université de Montrial, 2004

5(Ouvrages généraux:

-ADAMOV, Arthur, je....Ils, Paris, Gallimard, 1969

-BACHELARD, Gaston, L"eau et les rêves, Paris, LGF, 1993

-BOURDIN, Dominique, La psychanalyse de Freud à aujourd"hui, Paris, Breal,2000

$$
\begin{aligned}
& \text { العدد الرابع والعشرون (الجزء الثالث) } 2018 \\
& \text { مجلة كلية التربية- جامعة عين تمس55 }
\end{aligned}
$$


Quête de soi et dépression chez Albert Memmi

-CIRON, Emil,Syllogismes de 1"amertume, Paris, Gallimard,1952

-DOLTO, Françoise, La Solitude, Paris, Vertiges du Nord/Carrere,1987

-FREUD, Anna , Le Moi et les mécanismes de défense, Paris, Presses universitaires de France, 1946

-FREUD, Sigmand, La malaise dans la culture, Paris, Presses universitaires de France, 1995

-GROS,,Fréderic, Autrui, Paris, Hatier, 1999

-HEIDEGGER, Martin, L"être et le temps , Paris, Gallimard,1964

-KAHN,Axel , L"homme, ce roseau pensant, Paris, Nil, 2007

-MAALOUF, Amin, Les Identités meurtrières, Paris, Grasset,1988

-LOO, Henri \&LOO, Pierre, La dépression, Paris, Presses universitaire de France, 1991

-SARTRE, Jean Paul, L"être et le néant, Paris, Gallimard,1943

6(Ouvrages d'ordre narratologique et linguistique:

-REUTER,Yves, Introduction à l"analyse du roman, Pris, Bordas,1991

7(Dictionnaires

-CHEVALIER, Jean \& GHEERBRANT, Alain : Dictionnaire des symboles, Paris, Robert Laffont/ Jupiter, collec, Bouquins, 1986

-BLOCH, Henriette, CHEMAMA , Roland, DEPRET , Eric :Grand dictionnaire de la psychologie, Paris, Larousse, 1991

-REY, Alain :Le Petit Robert des noms propres, Paris, AMC, 1994

-COIN, Didier : Dictionnaire des symboles, des mythes et des légendes, Paris, Hachette, 2000

-MIJOLIA, Alain de : Dictionnaire International de la psychanalyse, Paris, Hachette, 2005

9 (Sites Internet:

-https//fr.m.wikipedia.org/Wiki/Wikip\%c3A9dia

:Accueilprincipal\#/languages

-http//psycha.ru//fr//dictionnaire/laplanche et pontalis/voc252html

https//www.jeuneafrique.com/63601/archives-thematiques/albert-memmila-d-colonisation-a-t-un-fruit-amer

-Heidegger, Martin : Être et temps, Éditions Numérique hors commerce 62 in t.m.p.free.fr $>$ Heidegger-être- et- temps

العدد الرابع والعشرون (الجزء الثالث) 2018

()

مجلة كلية التربية- جامعة عين شمس 


\section{Chahinaz Sabri Mohamed}

-Entretien d"Amin Maalouf avec Egi Volterrain, disponible sur le site d"Amin Maalouf ; voir aussi le revue Lire, Juin 2000, entretien avec Catherine Argand

-Bourget, Charles : «Les juifs à travers l"histoire » in Les dossiers d"histoire et de civilisation vol2, Cégep Scherbrooke, Canada,2008,p5

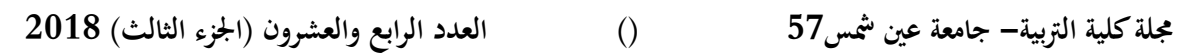


Quête de soi et dépression chez Albert Memmi

مجلة كلية الزبية- جامعة عين شمس58

